\title{
Nifedipine-Associated Acute Psychosis
}

JOEL K. KAHN, M.D.

Ann Arbor, Michigan

From the Department of internal Medicine, University of Michigan Medical School, Ann Arbor, Michigan. Reprints are not available. Manuscript submitted March 12, 1985 and accepted May 8, 1985.
A 84-year-old man was given nifedipine for control of angina pectoris. Acute psychosis with paranoid ideation developed soon after he began receiving the medication. All symptoms resolved with discontinuation of nifedipine. This is the second reported case of nifedipine-associated acute psychosis. Alternations in calcium-mediated neurotransmitter release, particularly dopamine, may be responsible. Clinicians should consider nifedipine when presented with a patient with acute psychosis.

The calcium channel antagonists are being used in a variety of vascular disorders. These agents have been used with remarkably few serious adverse effects [1], despite the fact that calcium regulation is vital in many biologic processes. Perturbations in calcium homeostasis have been described in several psychiatric disorders including mania, depression [2], and psychosis [3]; however, the calcium channel antagonists have rarely been associated with such disturbances $[4,5]$. This report describes a case of acute psychosis associated with nifedipine use, the second such case reported in the literature.

\section{CASE REPORT}

A 84-year-old man was admitted to the cardiology inpatient service for control of angina pectoris present for five months. In the weeks prior to admission, he had required increasing amounts of topical and sublingual nitroglycerin for pain relief. He had a poorly documented history of myocardial infarction a decade ago but was free of other medical or psychiatric problems. He denied symptoms of congestive heart failure or syncope. His medications included transdermal nitroglycerin $\left(20 \mathrm{~cm}^{2}\right)$ daily and sublingual nitroglycerin. Physical examination revealed an alert and pleasant man with a blood pressure of $118 / 82 \mathrm{~mm} \mathrm{Hg}$, a pulse of 50 per minute, and a respiratory rate of 18 per minute. He was afebrile. His lungs were clear. He had a grade 2/6 murmur characteristic of aortic stenosis but carotid upstrokes were normal. The results of neurologic examination and mental status were normal except for a moderate bilateral sensorineural hearing loss. Laboratory studies revealed normal electrolyte, urea nitrogen, creatinine, calcium, phosphorus, and transaminase values. Thyroid function results were normal. Urinalysis and chest roentgenography revealed no abnormalities. Electrocardiography shown sinus bradycardia with a rate of 50 per minute, first-degree atrioventricular block, left anterior fascicular block, and an old anteroseptal myocardial infarction. There were no recent changes on comparison with earlier tracings.

The patient was thought to have accelerated angina pectoris. Due to the electrocardiographic evidence of conduction system disease, he was given nifedipine ( $10 \mathrm{mg}$ four times a day) in addition to nitroglycerin paste ( 1 inch every four hours). While visiting with his family the day following admission, 
he made references to an airport that family members believed were out of context. Soon after their departure, he began insisting that he was in an airport and demanded his airplane tickets. He appeared agitated and was pacing restlessly. His vital signs were normal. When attempts were made to examine him, he accused the medical staff of trying to bring ruin on his family and causing him disgrace. $\mathrm{He}$ vehemently refused any further laboratory testing, claiming they were attempts by conspirators to do him harm. His family was contacted and returned immediately to remain with him overnight. The nifedipine was stopped. He persisted in accusations of conspiracy until he fell asleep that evening. The next morning, he was subdued but cooperative. In consultation with the psychiatry department, it was thought that he would benefit most by early discharge. $\mathrm{He}$ was discharged that morning, receiving isorbide dinitrate (10 $\mathrm{mg}$ four times daily) and sublingual nitroglycerin. Follow-up daily by phone and in the clinic one week later revealed that he had returned fully to his prior emotional state with adequate control of his angina pectoris.

\section{COMMENTS}

We present the second case of nifedipine-associated acute psychosis. The acute behavioral abnormalities in this patient were believed to be an adverse reaction to nifedipine for several reasons. This patient had no history of a psychiatric or dementing illness that may have spontaneously worsened. As he was a frequent world traveler, used to unfamiliar surroundings, it was unlikely that removal from his home environment was causative. Furthermore, his family was present when the abnormal behavior began. His confusion began during the daylight hours and therefore "sundowning" was unlikely. He had been using nitroglycerin on a long-term basis, and therefore nifedipine was the only new medication. Finally, the behavioral abnormalities reversed rapidly following discontinuation of the nifedipine.

Although the cause of psychotic disorders remains unknown, evidence suggests disturbances in neurotransmitter synthesis and release, particularly catecholamines. Excess dopaminergic activity has been suggested as a cause of psychosis [6]. Support for this hypothesis is derived from the observation that drugs that increase brain dopamine such as levodopa can induce a psychosis, and neuroleptic agents block dopaminergic activity in a fashion correlated with their anti-psychotic potency [6].

Calcium fluxes have been shown to be involved in neurotransmitter synthesis and release in nervous system tissue [2]. For example, calcium increases the activity of tyrosine hydroxylase, the rate-limiting enzyme in catecholamine synthesis, in noradrenergic neurons, whereas calcium chelation increases the activity of the enzyme in dopaminergic neurons [7]. Nifedipine acts upon channels that permit calcium passage through lipid membranes and is believed to plug such channels, inhibiting the influx of calcium [8]. Nifedipine may alter neurotransmitter synthesis and release through inhibition of calcium fluxes. In the central nervous system, calcium antagonism resulting in increased tyrosine hydroxylase activity in dopaminergic neurons would result in increased central dopaminergic activity, possibly altering behavioral patterns.

An alternative explanation is based on the observation that vincristine, a microtubule inhibitor, causes decreased levels of dopamine- $\beta$-hydroxylase in human plasma [9]. Calcium channel antagonists also have an effect on microtubular function [5], and their inhibition of dopamine- $\beta$ hydroxylase would increase the dopamine content of both dopaminergic and noradrenergic neurons in the central nervous system, again altering behavioral patterns.

This case presents an association between nifedipine and acute psychosis. Although use of the calcium channel antagonists has been relatively devoid of serious adverse effects [1], increasing clinical experience has resulted in the description of new clinical syndromes $[5,10]$. Clinicians should consider nifedipine along with other causes when faced with patients with acute psychosis.

\section{ACKNOWLEDGMENT}

I would like to thank Ms. Carol Cottrell for her secretarial assistance.

\section{REFERENCES}

1. Krebs R: Adverse reactions with calcium antagonists. Hypertension 1983; 5 (suppl II): II-125-II-129.

2. Dubovsky SL, Franks RD: Intracellular calcium ions In affective disorders: a review and an hypothesis. Biol Psychiatry 1983; 18: 781-797.

3. Carman JS, Wyatt RJ: Alterations in cerebrospinal fluid and serum total calcium with changes in psychiatric state. In: Usdin E, Hamburg DA, Barachas JD, eds. Neuroregulators and psychiatric disorders. New York: Oxford University Press, 1977; 488-494.

4. Ahmad S: Nifedipine-induced acute psychosis. J Am Geriatr Soc 1984; 32: 408.

5. Jacobs MB: Diltiazem and akathisia. Ann Intern Med 1983;
99: 794-795.

6. Van Kammen DP: The dopamine hypothesis of schizophrenia revisited. Psychoneuroendocrinology 1979; 4: 37-46.

7. Cooper JR, Bloom FE, Roth RH: The biochemical basics of neuropharmacology. New York: Oxford University Press, $1978 ; 175$

8. Braunwald $\mathrm{E}$ : Mechanism of action of calcium-channelblocking agents. N Engl J Med 1982; 307: 1618-1627.

9. Portugal MT, Vara F, Morales V, et al: Decrease in human plasma dopamine- $\beta$-hydroxylase levels with vinca alkaloid treatment. Biomedicine 1978; 29: 306-308.

10. Diamond JR, Cheung JY, Fang LST: Nifedipine-induced renal dysfunction. Am J Med 1984; 77: 905-909. 